\title{
A note on Gollier's model for a collective pension scheme
}

\author{
Johannes M. Schumacher* (D) \\ Amsterdam School of Economics, University of Amsterdam, Amsterdam, The Netherlands \\ ^Corresponding author. Johannes M. Schumacher: j.m.schumacher@uva.nl
}

(Received 9 August 2019; revised 9 January 2020; accepted 9 January 2020; first published online 7 February 2020)

\begin{abstract}
Gollier proposed in 2008 a model for the analysis of pension schemes that is helpful to focus attention on the impact of intergenerational risk sharing and on the role of the participation constraint. He uses the model to analyze the relative attractiveness of a collective scheme with respect to schemes that may be implemented by individuals for themselves. The analysis makes use of an assumption concerning the ownership rights of investment returns realized by generations that are between career start and retirement at the time of the transition from an individual to a collective system. The present paper investigates the consequences of adopting an alternative assumption. In a calibration exercise, the increase of the effective rate of return obtained by switching from an existing 'autarky' scheme to an infinite-horizon 'collective' scheme is found to be 8 basis points, as opposed to 72 basis points as reported by Gollier. Additionally, the effects are considered of changes in the specification of agents' preferences, aiming to express the specific nature of retirement income provision in the second pillar. The Black-Scholes assumptions are used to model the economic environment, so that many results can be obtained in closed form.
\end{abstract}

Key words: Autarky scheme; collective pension schemes; kinked utility; participation constraint; power utility; welfare analysis

JEL codes: G11; G22

\section{Introduction}

In the literature, many models have been proposed to support our understanding of the relative advantages and disadvantages of collective versus individual pension systems. Such models can be found, for example, in Gordon and Varian (1988), Krueger and Kubler (2006), Teulings and De Vries (2006), Ball and Mankiw (2007), Gollier (2008), Cui et al. (2011), Bovenberg and Mehlkopf (2014), and Chen et al. (2016). The variety of models used is, in part, a reflection of the manifold features of the problem that call for an investigation, such as intergenerational risk sharing, willingness to participate in collective schemes, redistributional effects, interaction with personal lifecycle planning, demographic developments, macroeconomic consequences, and so on. The model proposed by Gollier (2008) stands out as an elegant and highly stylized model, which serves well to focus attention on intergenerational risk sharing and on the participation constraint.

The present paper revisits the model of Gollier (2008) under several changes in assumptions. In particular, a modification will be investigated of the assumption in Gollier's paper relating to the ownership rights of retirement capital accumulated by generations that are between career start and retirement at the time at which a transition is made from an autarky system to a collective system. Furthermore, to express the preferences of agents, Gollier (2008) uses expected utility with respect to the classical CRRA utility function (constant relative risk aversion, i.e., power utility). The same framework will be used here as well, but additionally, two modifications to the CRRA utility functions 
will be investigated which both may be plausible from the point of view of second-pillar pension provision. In the first modification, a saturation level is imposed; second, a subsistence level (minimum payoff) is added. The preference specification is used in a welfare analysis with respect to several schemes that may be proposed. While Gollier (2008) labels the competing schemes as 'collective' and 'autarky', in this paper, the terms 'infinite-horizon' and 'moving-window' will be used. The terminology is meant to acknowledge that boundedness of the investment horizon is the key feature in what is called 'autarky' in Gollier (2008), and that the bound on the horizon is actually a parameter that may be varied. For low values of the bound, the scheme may be implemented in autarky, whereas a collective is needed to realize the same scheme in case the bound is higher than career length. The distinction between collective and autarky schemes is therefore not quite the same as the distinction between infinite-horizon and moving-window schemes. It would be incorrect to associate intergenerational risk sharing exclusively with the infinite-horizon scheme; such risk sharing is also required for movingwindow schemes with a window length that exceeds career length. In fact, although it will not be further analyzed in this paper, even under a shorter window length, the collective can still generate utility improvements when credit constraints prevent young generations from borrowing against future income.

The purpose of the paper is to contribute to the literature on the benefits of intergenerational risk sharing in pension systems. Specifically, fully funded systems are considered without external sponsoring, so that all contributions come from the participants themselves. The focus is on investment risk; in particular, sharing of longevity risk (clearly a beneficial feature of collective systems) is left out of the discussion. Within the context of an idealized financial model such as the Black-Scholes model that is used in this paper, the source of possible benefits of intergenerational risk sharing lies in the opportunity that it provides to individuals to obtain exposure to financial market risks during a time period that is longer than the period in which they would be able to invest on their own. The framework of Gollier (2008) is excellently suited to concentrate on this, abstracting away from the particulars of current pension schemes. ${ }^{1}$ Relative to the work of Gollier, the present paper contributes the following:

(i) reformulation in continuous time, so that more results become available in analytic form;

(ii) revision of the underlying assumptions concerning the ability of a social planner to reallocate the proceeds of investments of older generations, and explicit discussion of financial fairness;

(iii) recalculation of the benefits of intergenerational risk sharing under the revised assumptions;

(iv) inclusion of schemes that are neither of collective nor of autarky type in Gollier's terminology, i.e., moving-window schemes with a window length that exceeds career length;

(v) inclusion of non-CRRA utilities.

Under the revised assumptions, it turns out that the utility gains of replacing an existing 40-year moving-window scheme by an infinite-horizon scheme are not nearly as large as calculated in Gollier's paper. The comparison can be tilted in favor of the infinite-horizon scheme by excluding a number of the generations closest to retirement from the transfer to the new scheme. The utility gains of a moving-window scheme increase with the length of the investment window, and generations will prefer an existing moving-window scheme to the introduction of an infinite-horizon scheme if the window length is sufficiently large. However, under modifications of the CRRA preferences that can be justified from a second-pillar perspective, the rate of improvement due to horizon lengthening decreases for longer horizons. Moreover, it has to be taken into account that there is a downside of horizon lengthening, which consists in the risk that a future incoming generation will be faced with a deficit on its pension savings account. It is up to policy makers to balance the pros and cons; the present paper just aims to display the tradeoffs and to highlight the impact of key parameters such as the interest rate, the market price of risk, and the degree of risk aversion of scheme members.

\footnotetext{
${ }^{1}$ This also means that the analysis is set in a partial-equilibrium framework, and that other pathways for intergenerational risk sharing, such as national debt, are left out of consideration. A survey of the literature on intergenerational risk sharing that includes these aspects is available in Beetsma and Romp (2016).
} 
The paper is organized as follows. Section 2 specifies the model and details the modifications with respect to Gollier (2008). In Section 3, the CRRA utility function used by Gollier is maintained; the investigation focuses on the consequences of the change in assumptions concerning the investments of older generations for the relative attractiveness of various schemes. Subsequently, in Section 4, an analogous study is carried out under the modifications of the CRRA utility function as indicated above. Conclusions follow in Section 5. There is an Appendix containing derivations of formulas used in the main body of the paper.

\section{Outline of the model}

\subsection{Modifications with respect to Gollier (2008)}

The model of Gollier (2008) may be described briefly as follows. A collective pension fund is formed at time $t=0$. The fund collects contributions from participating generations, invests the received income in the financial markets, and pays benefits to participating generations after retirement. Inflation is set to zero; this entails no loss of generality, since equivalently one can use the convention of expressing all monetary quantities in inflation-indexed units. Contributions are constant (as measured in inflation-indexed units). The size of the benefits is not determined in advance, and may depend on investment returns. In the economy in which the fund operates, there is a single risky asset as well as a riskless asset. Returns on the risky asset are independent and identically distributed. The annual return on the riskless asset is a fixed number $r_{f}$, which, under the conventions stated above, denotes the discretely compounded real interest rate. The collective system is assumed to remain in existence forever, so that the present value of future contributions can be included in the initial capital. The fund is free to choose an investment policy. Benefits are paid to retiring generations as a lump sum at the moment of retirement. Each year, one generation retires, and all generations are equally large.

The analysis of Gollier (2008) focuses on the welfare improvements that can be achieved by switching, at time $t=0$, from a system of 'autarky', in which people save for their own retirement, to a 'collective' system as described above. For a proper comparison, the size of pension contributions paid by individuals in both systems is supposed to be the same. It is assumed that borrowing against future income is possible, so that, in the autarky system, the full present value of pension contributions is available for investment already at the start of an individual's working career.

An important issue is the treatment of the working generations, i.e., the generations that, at the time of the reform, are between career start and retirement. These generations have already started investments to support retirement income, and may have experienced gains or losses. When a transition is made to a collective system, on what basis will these generations participate? Computations in the present paper will be based on the following procedure, which could be replaced by any other rule that is equivalent to it from an amortization point of view (i.e., payments could be shifted in time, with due discounting based on the risk-free rate $r_{f}$ ). At the start of the new system, the working generations first pay back the loans they have taken out, so that they enter the new system with a clean slate. After that, they make a full contribution to the collective fund, and in return receive a full benefit when they retire. They are not required to bring in the gains that they may have enjoyed from earlier investments, nor are they entitled to compensation from the collective scheme in case these investments have underperformed with respect to a riskless savings account.

The point of view above is different from the one used in Gollier (2008). In that paper, it is assumed that the full amount of the retirement savings accumulated under autarky will flow into the assets of the collective fund. A priori, the size of these savings is a random variable; it depends on the extent to which pension savings have been invested in risky assets, and on the returns on risky assets that the working generations have experienced. It is assumed in Gollier's paper that working generations have followed a lifecycle plan that is optimal under the preference specification used in the paper, and that the outcome of the investments is equal to the expectation (under the real-world measure) of the results generated by this plan. Since the expected investment return is higher than the riskless rate, this creates an additional inflow into the capital available to the collective fund with respect to the assumptions made here. 
The present paper will moreover use a different assumption concerning the schedule of payments. This is, however, just a matter of convenience, based on the equivalence of amortization schedules that is easily established under the assumption of constant interest rates. The schedule that is assumed in Gollier (2008) for the time after the transform is that generations pay an equal amount each year until the time of retirement. In this paper, the payments by generations are handled as if they are transferred as a lump sum at the time of retirement. The assumption that generations receive their pension benefit and pay their contribution at the same time, both as a lump sum, makes it easy to treat all participating generations in the same way, irrespective of whether they are already working at the time of transform or will enter later.

Of course, proper discounting needs to be applied to align the two payment schedules. The schedule with annual contributions can be converted to a schedule with a lump sum contribution at the time of retirement by assuming that generations deposit their annual contribution $y$ in a riskless savings account, and that they close the account at the time of retirement in order to pay their contributions. Since one generation retires per year, the institution then receives $\sum_{j=1}^{n}\left(1+r_{f}\right)^{j} y$ each year. If, on the other hand, the $n$ generations that are active in any given year pay the amount $y$ directly to the institution, as assumed in Gollier's paper, then the institution each year receives $n y$.

In the concrete numerical example of Gollier (2008), the interest rate $r_{f}$ is $2 \%$, and the number of contemporaneous working generations is $n=40$; this also means that generations pay contributions during 40 years. The total time- 0 value of contributions received by the fund starting from the time of the reform is therefore $\left(\left(1+r_{f}\right) / r_{f}\right) n y=2040 y$. To this, the expected value of the investments of currently working generations is added, which is equal to $1638 y$ according to the calculations of Gollier (2008). Under the assumptions of the present paper, the initial capital available to the pension institution at the time of the transform is equal to the time- 0 value of a stream of annual payments of size $C=\sum_{j=1}^{n}\left(1+r_{f}\right)^{j} y$. Subject to the parameter values as given, the size of the annual income of the fund is $61.61 y$, and the corresponding time- 0 value of the annual income stream is $3142 y$. With respect to the value $3678 y$ cited by Gollier, this is a $14.6 \%$ reduction.

\subsection{Formulation of schemes}

Consider the time- 0 situation of a fund that will receive lump sum contributions of size $C$ at times $t=$ $1,2, \ldots$ and that will pay lump sum retirement benefits at the same instants of time. ${ }^{2}$ The total net present value of the assets available to the collective scheme at time 0 is given by

$$
A_{0}=\sum_{\infty}^{j=1}\left(1+r_{f}\right)^{-j} C=\frac{1}{r_{f}} C .
$$

This may also be stated by saying that the contribution required from the generations to make the total time- 0 value of available assets equal to $A_{0}$ is $C=r_{f} A_{0}$. It is assumed that the fund is not credit constrained and that it can freely choose both an investment policy and a benefit policy, subject only to the constraint that the total time- 0 value of paid benefits should not exceed the total time- 0 value of contributions.

The benefits are in general not deterministic; in particular, they may depend on investment results. Given a preference model, one may define the certainty equivalent of the benefit received by a given generation as the amount such that the generation is indifferent between receiving either this deterministic amount or the benefit from the pension system. Gollier (2008) assumes that all generations use the same preference model. Given a benefit policy, one can then compute the certainty equivalent for each generation. Gollier proposes as a design principle that this certainty equivalent should be the same for all generations. An outline of pension system design may then be written as follows. The term 'generation $j(j=1,2 \ldots)$ is used to refer to the generation that will retire at time $j$.

\footnotetext{
${ }^{2}$ Contributions and benefits at time 0 are not included, in contrast to the previous section; this slightly simplifies the formulas.
} 


\section{Infinite-horizon scheme}

(i) Start from a given level of contributions $C$ (lumped to time of retirement).

(ii) For each generation $j$, determine the function that, for a given number $C E$, produces the minimal amount of capital at time 0 needed to realize a payoff at the time of retirement $T_{j}$ that has $C E$ as its certainty equivalent. Let this function be denoted by $v_{0}(C E, j)$. Also determine the corresponding investment policy.

(iii) Solve for $C E$ from the equation

$$
\frac{1}{r_{f}} C=\sum_{j=1}^{\infty} v_{0}(C E, j) .
$$

(iv) Form the fund's investment policy as the sum of the investment policies for each of the separate generations. Pay to each generation the result of the investments that were made on its behalf.

In this calculation, the level of contributions is taken as a starting point, and the certainty equivalent of pension benefits (which is the same for all generations) is an output. If participants are not satisfied with the level of the certainty equivalent, they may choose to raise the level of contributions. Depending on the preference model that is used, the quantities $v_{0}(C E, j)$ may or may not depend linearly on $C E$; examples of nonlinear dependence will appear below.

Step (ii) in the procedure above reverses the standard formulation of optimization problems, in which initial capital is given and one looks for the highest certainty equivalent that can be realized. Of course, this is just a reformulation and the same solution techniques can be used, be it that iteration may be required in cases where solutions are not available in sufficiently analytical form.

The leading subject of discussion in Gollier's paper is a comparison of the scheme that results from the above with an alternative scheme that is labeled 'autarky'. In the alternative scheme, generations invest for themselves starting from the beginning of their working careers. The design of this scheme may be summarized as stated below. The investment horizon will be denoted by the symbol $\tau$. If individuals can borrow against future income, then $\tau$ is equal to career length, but the effective length of the investment horizon is shorter if credit constraints are active. On the other hand, the same scheme with a longer investment window could be realized within a collective fund. The scheme will therefore in this paper not be labeled as an 'autarky' scheme but rather as a 'moving-window' scheme. It will be assumed that the nature of the surrounding economy does not change over time, so that all generations face the same investment opportunities.

\section{Moving-window scheme}

(i) Start from a given level of contributions $C$ (lumped to time of retirement).

(ii) Fix an investment window length $\tau$.

(iii) Find the optimal certainty equivalent $C E$ that can be generated at time $T$ on the basis of available capital $e^{-r \tau} C$ at time $T-\tau{ }^{3}$ Under the stated assumption of time-homogeneity of the economy, the optimal certainty equivalent depends on $\tau$ but not on $T$. Also determine the corresponding optimal investment policy.

(iv) Implement the investment strategy for each generation $j$, starting at time $T_{j}-\tau$. Pay to each generation the result of the investments that were made on its behalf.

The two schemes differ in several respects:

- investment horizon: in the infinite-horizon scheme, the investment horizon varies from 1 for the first retiring generation to arbitrarily large values for later generations, whereas the investment horizon in the moving-horizon scheme is fixed at the value $\tau$;

\footnotetext{
${ }^{3}$ The letter $r$ denotes the continuously compounded interest rate that corresponds to the discretely compounded rate $r_{f}$.
} 
- fairness: the infinite-horizon scheme is fair from the welfare perspective, while the movingwindow scheme is fair both from the welfare perspective and from the valuation point of view;

- implementability: the infinite-horizon scheme can only be implemented by a collective system, whereas the moving-window scheme can be implemented in autarky if the investment window $\tau$ is not too large.

In the above, the term 'fair from the welfare perspective' means that all generations enjoy the same level of expected utility, or in other words that the certainty equivalents of their payoffs (as viewed from time 0) are the same, whereas 'fair from the valuation point of view' indicates that the ex-ante market values of their payoffs are the same, which also implies that the market value of the contribution of each generation is equal to the market value of the benefit it receives. The term 'not too large' in relation to the investment window means, in case borrowing against future income is possible, that the window length should not be larger than career length.

The time- 0 value of the contingent benefit received by generation $j$ is equal to $v_{0}(C E, j)$ in the infinite-horizon scheme, where $C E$ is defined by (2), whereas in the case of the moving-window scheme, the time- 0 value is $e^{-r \tau} C=\left(1+r_{f}\right)^{-j} C$. In both cases, the total net present value of benefits is equal to $C / r_{f}$, which is the total net present value of contributions. The value allocated to any generation can therefore not be increased without decreasing the value allocated to at least one of the other generations. Under the assumption (which is typically satisfied) that the relation between value on the one hand and optimal certainty equivalent on the other hand is strictly increasing, this implies that the infinite-horizon scheme is Pareto efficient as seen from time 0; the same holds for the moving-horizon scheme, subject to the constraint that the benefit paid at time $T$ is contingent only upon asset returns in the interval between $T-\tau$ and $T$.

Both in the infinite-horizon scheme and in the moving-window scheme with window length exceeding career length, a decision is taken at time $t=0$ that affects generations that are not yet active at that time. ${ }^{4}$ Specifically, investments are already started at their behalf before these generations enter the labor force. The interests of these future generations must be safeguarded. It will be assumed in this paper that a policy decision at time $t=0$ concerning the choice of a system for retirement savings needs the consent of all generations, whether they are actually present or only virtually. To ensure unanimity, schemes will be constructed such that the certainty equivalents of benefits, as seen from time $t=0$, are the same for all generations; this is in agreement with the fairness constraint imposed in Gollier (2008, p. 1472). Moreover, generations that are not active yet at time 0 are supposed to be free to decide at the start of their working careers whether or not they will participate in the scheme. Under the assumption that these generations can implement for themselves a moving-window scheme with window length equal to career length, ${ }^{5}$ future generations will rationally decide not to participate if the investments that were made on their behalf, prior to the start of their working careers, have led to a deficit. This is the participation constraint. Possible mitigation of the restrictions imposed by this constraint by use of a risk-bearing entity from outside is discussed in Gollier (2008). In this paper, the analysis will be limited to providing expressions for the probability that the participation constraint will be triggered within a given scheme.

An underlying assumption in the analysis is that the expected utility of benefits paid by the fund is indeed a good way of measuring the welfare of generations. Even if one agrees to concentrate only on retirement income as a factor in welfare and accepts that there is a utility function that allows expression of preferences by means of expected utility, it can still be argued that welfare in retirement should be measured by total income, of which the benefit paid by the fund is only a part. Additional effective sources of income may relate for instance to private savings, to home ownership, and to financial and in-kind support by relatives. In line with most of the literature, these alternative sources will be ignored

\footnotetext{
${ }^{4}$ This is not unusual by itself; in fact, such decisions are being made constantly, for instance regarding national debt, infrastructure, environmental policy, and so on.

${ }^{5}$ In particular, this requires that it is possible for individuals to borrow against future income.
} 
and comparison between the two schemes will be based purely on the expected utility associated with the benefits that these schemes produce.

\subsection{Economic assumptions}

The preference models used in this paper are all of the expected utility type. ${ }^{6}$ The classical model with constant relative risk aversion will be used, as in Gollier's paper, but variations that incorporate a level of saturation and a guaranteed minimum level will be investigated as well. Concerning the economic environment, the Black-Scholes model will be used throughout. The price of risk associated with the single risk factor in the BS economy is denoted by $\lambda$. A portfolio in the BS economy that has constant volatility $\sigma$ must follow a stochastic differential equation of the form

$$
d S_{t}=(r+\lambda \sigma) S_{t} d t+\sigma S_{t} d W_{t} .
$$

The growth optimal portfolio denoted by $G_{t}$ is the portfolio with volatility $\lambda$, so that

$$
d G_{t}=\left(r+\lambda^{2}\right) G_{t} d t+\lambda G_{t} d W_{t}
$$

It is well known that the growth optimal portfolio is the inverse of the pricing kernel, or, in other words, the real-world measure $P$ can be used as the pricing measure if the growth optimal portfolio is used as the numéraire. The pricing measure that corresponds to taking the money market account as the numéraire will be denoted by $Q$. Both the continuous-time real interest rate $r$ and its discrete-time equivalent $r_{f}=e^{r}-1$ will be used in this paper. It is assumed throughout that both $r$ and $\lambda$ are positive.

The main preference model used in this paper is the same as the one used in Gollier (2008), namely expected utility under the classical CRRA utility function

$$
u(x)=\frac{x^{1-\gamma}}{1-\gamma}
$$

It is a standard fact (see for instance Merton (1990)) that the corresponding optimal payoff ('benefit') $B_{T}$ at time $T$ that can be realized subject to a budget constraint $E^{Q} B_{T}=v$ is of the form

$$
B_{T}=\alpha G_{T}^{1 / \gamma}
$$

where $\alpha$ is a constant that must be chosen to satisfy the budget constraint. This payoff is realized by a portfolio of constant volatility $\lambda / \gamma$. The expected excess return of this portfolio is equal to $\lambda^{2} / \gamma^{7}$ In numerical exercises, the following parameter values will be used:

$$
r=0.02, \quad \lambda=0.287, \quad \gamma=5 \text {. }
$$

The value for the risk premium $\lambda$ is computed as $(\mu-r) / \sigma$ with expected real excess return $\mu-r=$ 0.039 and volatility $\sigma=0.136$ as reported in Gollier (2008, p. 1468). The values of $r$ and $\sigma$ are also taken from the same paper.

\footnotetext{
${ }^{6}$ This is not to say, of course, that other models would not be interesting to consider as well.

${ }^{7}$ In this paper, the expected excess return at time $t$ of a portfolio with value $V_{t}$ at time $t$ is defined as $\lim _{\Delta t \downarrow 0}\left(e^{-r \Delta t} E\left[V_{t+\Delta t} /\right.\right.$ $\left.\left.V_{t}\right]-1\right) / \Delta t$. In other words, this is the expected value of the arithmetic return minus the riskless rate.

${ }^{8}$ The discrete-time parameter values as reported by Gollier are used here as continuous-time parameter values. Consequently, a model is obtained that is only approximately the same as the model used in numerical exercises by Gollier. An exact match between the two models does not seem possible, since in the Black-Scholes model, returns are distributed lognormally, whereas Gollier apparently assumes (it is not stated explicitly in his paper) that they follow a normal distribution.
} 


\section{Constant relative risk aversion}

In this section, the welfare implications of various schemes are investigated under the assumption of CRRA preferences. A few alternative preference specifications are discussed in Section 4. There are two subsections. In the first, the choice between competing schemes is discussed from a fixed point in time at which a transition from a moving-window scheme to an infinite-horizon scheme is contemplated. This implies that a decision is made partly on behalf of future generations. In the second subsection, the probability is investigated that those generations will indeed take the same decision, when they make use of information that has become available since the start of the scheme.

\subsection{Choosing between schemes at time 0}

\subsubsection{The relation between contribution and certainty equivalent}

The certainty equivalent of the payoff $\alpha G_{T}^{1 / \gamma}$ appearing in (6) and the capital required at time 0 to generate this payoff are given, respectively, by

$$
\begin{gathered}
C E=\alpha G_{0}^{1 / \gamma} \exp \left(\frac{1}{\gamma}\left(r+\frac{1}{2} \lambda^{2} / \gamma\right) T\right) \\
v_{0}=\alpha G_{0}^{1 / \gamma} \exp \left(-\frac{\gamma-1}{\gamma}\left(r+\frac{1}{2} \lambda^{2} / \gamma\right) T\right) .
\end{gathered}
$$

From (8) and (9), one finds the capital needed at time 0 to produce the certainty equivalent $C E$ at time $T$ :

$$
v_{0}(C E, T)=e^{-\left(r+\frac{1}{2} \lambda^{2} / \gamma\right) T} C E .
$$

For each given horizon length $T$, this formula establishes a linear relationship between time- 0 value on the one hand and certainty equivalent on the other. The linear nature of the relation between certainty equivalent and required capital is a specific feature of CRRA utility; under the modified utility specifications that will be discussed in Section 4 below, the relation between the two quantities is nonlinear.

Relating to the dependence on horizon length, the formula (10) implies that the amount needed at time 0 to create a given level of utility for a beneficiary at time $T$ decreases with increasing horizon length at a rate that is faster than the amount needed at time 0 to generate a given fixed capital at time $T$. This may be explained as an effect of increased diversification. Agents with a longer investment horizon are exposed to a longer history of asset returns, which gives them an advantage, at least as seen from the time at which investments are started, with respect to agents who take out their benefits at an earlier time. More specifically, the formula shows that, under CRRA utility in a BS economy, advancing the start of investment by a time interval of length $L$ reduces the funds required to reach a given level of utility by the factor exp $\left(-\left(\frac{1}{2} \lambda^{2} / \gamma\right) L\right)$. This relationship is already implicitly contained in the work of Merton (1969). In other words, $\frac{1}{2} \lambda^{2} / \gamma$ is the 'bonus interest rate' that is received (from a utility point of view) by accepting a certain amount of risk.

Earning this bonus rate is the key reason for the lengthening of the investment horizon, as realized by intergenerational risk sharing. The quantity $\frac{1}{2} \lambda^{2} / \gamma$ will consequently make a frequent appearance in this paper. Several formulas below are simplified by introducing

$$
r_{d}:=e^{r+\frac{1}{2} \lambda^{2} / \gamma}-1
$$

which is the discrete-time equivalent of $r+\frac{1}{2} \lambda^{2} / \gamma$, in the same way as $r_{f}$ is the discrete-time equivalent of the interest rate $r$. 


\subsubsection{Infinite-horizon scheme}

In the case of the infinite-horizon scheme, the benefit that will be received by generation $j$ (i.e., the generation that will retire at time $T_{j}=j$ ) can be computed as follows. Given the relation (10), equation (2) that expresses equality between available capital and required capital can be written more explicitly as follows:

$$
\frac{1}{r_{f}} C=\sum_{j=1}^{\infty} e^{-\left(r+\frac{1}{2} \lambda^{2} / \gamma\right) T_{j}} C E=\frac{1}{r_{d}} C E,
$$

where $r_{d}$ is defined in (11). Hence, the certainty equivalent that is achieved by the infinite-horizon scheme based on the contribution level $C$ is given by

$$
C E_{\mathrm{ih}}=\frac{r_{d}}{r_{f}} C
$$

Therefore, the ratio $r_{d} / r_{f}$ (which is always at least equal to 1) represents the bonus of intergenerational risk sharing via the infinite-horizon scheme. From the value given by (13), one can compute the factor $\alpha$ in the expression (6) either via (8) or via (10) and (9). One finds that the benefit received under the infinite-horizon scheme by the generation that retires at time $T$, given the lump sum contribution $C$, is equal to

$$
B_{T}^{\mathrm{ih}}=\left(\frac{G_{T}}{G_{0}}\right)^{1 / \gamma} \exp \left(-\frac{1}{\gamma}\left(r+\frac{1}{2} \lambda^{2} / \gamma\right) T\right) \frac{r_{d}}{r_{f}} C .
$$

These benefits for different values of $T=T_{j}$ are not equally distributed; nevertheless, by construction, their certainty equivalents under the CRRA utility function are the same. Some characteristics of interest are:

$$
E\left[B_{T}^{\mathrm{ih}}\right]=\exp \left(\left(\frac{1}{2} \lambda^{2} / \gamma\right) T\right) \frac{r_{d}}{r_{f}} C, \quad \operatorname{std}\left(B_{T}^{\mathrm{ih}}\right)=E\left[B_{T}^{\mathrm{ih}}\right] \sqrt{e^{\left(\lambda^{2} / \gamma^{2}\right) T}-1}
$$

As noted in Gollier (2008), both of these quantities are increasing in T. One can also compute the probability that the benefit at time $T$ is at least equal to the contribution made by the generation that receives it: ${ }^{9}$

$$
P\left(B_{T}^{\mathrm{ih}} \geq C\right)=\Phi\left(\frac{1}{2} \frac{\gamma-1}{\gamma} \lambda \sqrt{T}+\frac{\gamma}{\lambda \sqrt{T}} \log \frac{r_{d}}{r_{f}}\right)
$$

A plot of this probability as a function of the time of payment of the benefit (measured in years after the implementation of the scheme) is shown in the left panel of Figure 1. The figure shows results under the calibration $\gamma=5$. For lower values of $\gamma$, the fund invests less conservatively, which increases both expected return and volatility; the volatility effect dominates, resulting in lower values of the probability of at least matching the value of the contribution when $\gamma$ is decreased. For $\gamma=1$, it is seen from (16) that the probability tends to 0.5 for long horizons. The time- 0 value of the benefit

\footnotetext{
${ }^{9}$ Note that these quantities can be compared directly, since the contribution is lumped to the time at which the benefit is paid. Stated differently, the quantity $C$ can be interpreted as the retirement capital that would have been built up by a given generation if it would have invested all of its pension contributions in riskless assets.
} 

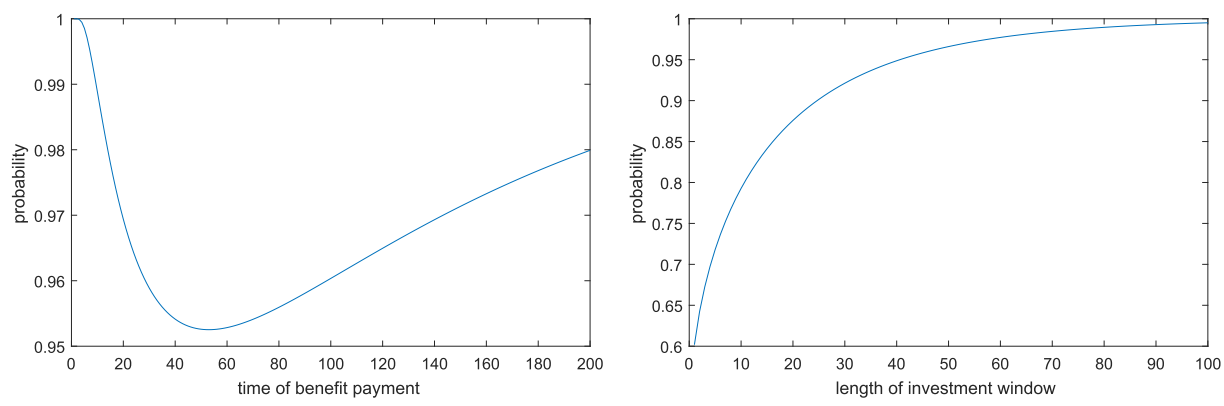

Figure 1. The figure shows the probability that the benefit received is at least equal to the contribution paid in the case of the infinite-horizon scheme (left panel) and in the case of the moving-window scheme (right panel). In the first case, the probability is given as a function of the time at which the benefit is paid, as measured from the time at which the infinite-horizon scheme is initiated. In the case of the moving-window scheme, the probability is shown as a function of window length. Parameter values are as in (7).

paid at time $T_{j}$ is

$$
v_{0}\left(B_{j}^{\mathrm{ih}}\right)=\left(1+r_{d}\right)^{-j} \frac{r_{d}}{r_{f}} C=\left(1+r_{d}\right)^{-j} r_{d} A_{0}
$$

where $A_{0}:=C / r_{f}$ is the initially available capital, namely the total net present value of contributions. The time- 0 values of the benefits for $j=1,2, \ldots$ add up to the initial capital $A_{0}$, as it should be the case.

\subsubsection{Moving-window scheme}

In the case of the moving-window scheme, an explicit expression for the pension benefits can be found as follows. From the expression (10), the capital required at time $T-\tau$ to generate the certainty equivalent $C E$ at time $T$ is equal to $e^{-\left(r+\frac{1}{2} \lambda^{2} / \gamma\right) \tau} C E$. The relation under the moving-window scheme between the certainty equivalent and the contribution level is therefore given by

$$
C E_{\mathrm{mw}}=e^{\left(\frac{1}{2} \lambda^{2} / \gamma\right) \tau} C=\left(\frac{1+r_{d}}{1+r_{f}}\right)^{\tau} C
$$

Consequently, the quantity $\left(\left(1+r_{d}\right) /\left(1+r_{f}\right)\right)^{\tau}$ represents the bonus of intergenerational risk sharing via the moving-window scheme. The parameter $\alpha$ in the expression (6) can again be determined either via (8) or via (10) and (9). From this, one finds that the benefit received by generation that retires at time $T$ under the moving-window scheme, given the lump sum contribution $C$, is

$$
B_{T}^{\mathrm{mw}}=\left(\frac{G_{T}}{G_{T-\tau}}\right)^{1 / \gamma} \exp \left(-\frac{1}{\gamma}\left(r-\frac{1}{2} \lambda^{2}(\gamma-1) / \gamma\right) \tau\right) C .
$$

Under the assumption of time-homogeneity of the economic environment, these random variables are all identically distributed. One has

$$
E\left[B_{T}^{\mathrm{mw}}\right]=e^{\left(\lambda^{2} / \gamma\right) \tau} C, \quad \operatorname{std}\left[B_{T}^{\mathrm{mw}}\right]=E\left[B_{T}^{\mathrm{mw}}\right] \sqrt{e^{\left(\lambda^{2} / \gamma^{2}\right) \tau-1}}
$$

The probability that the benefit received by a generation will be at least as large as the contribution that 
it pays is given by

$$
P\left(B_{T}^{\mathrm{mw}} \geq C\right)=\Phi\left(\frac{\gamma-\frac{1}{2}}{\gamma} \lambda \sqrt{\tau}\right)
$$

A plot of this probability as a function of the window length $\tau$ is shown in the right panel of Figure 1 . As in the case of the infinite-horizon scheme, the probability goes down when $\gamma$ is decreased. The time- 0 value of the benefit paid at time $T_{j}$ is

$$
v_{0}\left(B_{j}^{\mathrm{mw}}\right)=\left(1+r_{f}\right)^{-j} C=\left(1+r_{f}\right)^{-j} r_{f} A_{0} .
$$

By construction, the sum of the time- 0 values of the benefits for $j=1,2, \ldots$ equals the initial capital $A_{0}$.

\subsubsection{Comparison}

Now, let us consider, as in Gollier's paper, the situation at time 0 when generations decide whether or not to move from an existing moving-window scheme to an infinite-horizon scheme. Preference for one scheme or the other is determined for all generations by comparing (13) to (18). It is seen that, under the CRRA utility function, the choice between the two does not depend on the contribution level. The infinite-horizon scheme will be preferred to the moving-window scheme by all generations at time 0 if $r_{d} / r_{f}>\exp \left(\left(\frac{1}{2} \lambda^{2} / \gamma\right) \tau\right)$, or in other words if $\tau<\tau_{\text {crit }}$ where the 'critical window length' $\tau_{\text {crit }}$ is defined by

$$
\tau_{\text {crit }}=\frac{\log r_{d}-\log r_{f}}{\frac{1}{2} \lambda^{2} / \gamma} .
$$

Under the parameter values given by (7), one has $\tau_{\text {crit }}=42.37$ years. At the window length $\tau=40$ that is assumed in Gollier (2008), the infinite-horizon scheme is preferred to the moving-window scheme, though not by a wide margin. The comparison may also be stated in terms of equivalent savings rates, which in the continuous-time framework are defined by the equations

$$
\frac{r_{d}}{r_{f}} \frac{e^{r T}-1}{r}=\frac{e^{r_{\mathrm{ih}} T}-1}{r_{\mathrm{ih}}} \quad \text { and } \quad e^{\left(\frac{1}{2} \lambda^{2} / \gamma\right) \tau} \frac{e^{r T}-1}{r}=\frac{e^{r_{\mathrm{mw}} T}-1}{r_{\mathrm{mw}}}
$$

for the infinite-horizon case and the moving-window case, respectively; the number $r_{\text {ih }}$ is the interest rate that, when applied to a savings scheme with the same annual contributions, leads to a final capital that agrees with the certainty equivalent obtained from the infinite-horizon scheme, and $r_{\mathrm{mw}}$ has the same interpretation with respect to the moving-horizon scheme. Under the parameter assumptions (7), one finds from (24): $r_{\mathrm{ih}}=3.48 \%, r_{\mathrm{mw}}=3.40 \%$. The corresponding values in Gollier (2008) are (see p. 1473 and p. 1468) $4.05 \%$ and $3.33 \%$. In the moving-horizon case, the difference is due to the use of discrete versus continuous time, as discussed in footnote 8 , while the difference in the infinite-horizon case is largely due to the different assumption made in this paper regarding the ownership rights of the pension savings of generations in the labor force at $t=0$. The advantage of 0.72 percentage points of the infinite-horizon scheme over the moving-horizon scheme with a window length of 40 years, as reported by Gollier, is therefore seen to be reduced to 0.08 percentage points under the revised assumptions.

Of course, these conclusions depend on the values of the parameters in the model. Table 1 shows critical window lengths for a range of different values of the real interest rate $r_{f}$ and the parameter $\lambda^{2} / \gamma$, which represents the expected excess return of the optimal portfolio held by a CRRA investor with risk 
Table 1. Critical window lengths

\begin{tabular}{lcccccr}
\hline & \multicolumn{7}{c}{ Expected excess return $\lambda^{2} / \gamma$} \\
\cline { 2 - 7 }$r(\%)$ & $1 \%$ & $2 \%$ & $3 \%$ & $4 \%$ & $5 \%$ & $6 \%$ \\
\hline 0.5 & 139.13 & 110.36 & 92.92 & 80.97 & 72.17 & 65.37 \\
1.0 & 81.59 & 69.82 & 61.59 & 55.43 & 50.61 & 46.71 \\
1.5 & 58.04 & 51.58 & 46.71 & 42.86 & 39.74 & 37.12 \\
2.0 & 45.13 & 41.05 & 37.81 & 35.16 & 32.94 & 31.05 \\
2.5 & 36.97 & 34.15 & 31.84 & 29.89 & 28.23 & 26.79 \\
\hline
\end{tabular}

A moving-window scheme with a window length that is larger than this critical value will at time 0 be preferred by all generations to an infinite-horizon scheme.

For a range of values of the real interest rate $r$ and the expected excess return $\lambda^{2} / \gamma$, the table shows the corresponding critical window length.

aversion parameter $\gamma \cdot{ }^{10}$ It is seen that the infinite-horizon scheme is preferred in particular in situations in which the interest rate is low, ${ }^{11}$ and the parameter $\lambda^{2} / \gamma$ is low as well. ${ }^{12}$

It may be surprising to note that, under some parameter values, generations will unanimously prefer a moving-window scheme to an infinite-horizon scheme. The relation (10) suggests that one should always start investing as early as possible. Why should future generations then not be happier with a scheme that already starts investing for them at time 0 , long before they are even born, than with a scheme that limits them to a fixed investment horizon? The answer lies in the fact that the infinite-horizon scheme redistributes wealth between generations. For a fixed initial capital $A_{0}$ (equivalently, a fixed level of contributions), the time- 0 values of benefits of generations under the infinite-horizon scheme and under the fixed-window scheme are given, respectively, by $v_{0}\left(B_{j}^{\mathrm{ih}}\right)=r_{d}\left(1+r_{d}\right)^{-j} A_{0}$ and $v_{0}\left(B_{j}^{\mathrm{mw}}\right)=r_{f}\left(1+r_{f}\right)^{-j} A_{0}$, as shown in (17) and (22). Since $r_{d}>r_{f}$, we have $v_{0}\left(B_{j}^{\text {ih }}\right)>v_{0}\left(B_{j}^{\mathrm{mw}}\right)$ for low values of $j$, whereas the reverse inequality holds for high values of $j$; the crossover point is at the critical window length $\tau_{\text {crit }}$. In other words, the infinite-horizon scheme transfers value from later generations to earlier generations. On the other hand, the construction of the moving-window scheme is such that the time- 0 value of the benefit received by each generation is equal to the time- 0 value of its contribution. This is the principle of 'generational accounting' propounded in Teulings and De Vries (2006), sometimes also called 'financial fairness'. The value transfer in the infinite-horizon scheme is motivated from a welfare point of view. Later generations benefit from the fact that investments on their behalf have already been made at what is for them a very early time, whereas the absence of this advantage for the earlier generations justifies that they should be compensated. Nevertheless, later generations may feel that they are better off in the moving-window scheme, in which investments on their behalf are made on a shorter horizon, but on the basis of the full value of their contribution. By the fact that certainty equivalents are by construction equal to each other, this happens exactly in situations in which the earlier generations feel that the value compensation they receive from the infinite-horizon scheme is not sufficient. If one would try to appease later generations by shifting less value away from them, the result would necessarily be that earlier generations become even more unhappy than they already are, so that agreement could still not be reached.

\footnotetext{
${ }^{10}$ While the appropriate values of the price of risk $\lambda$ and the coefficient of risk aversion $\gamma$ separately are subject to much debate, it may be said that, under the perhaps rather bold assumption that pension funds act like CRRA investors, direct empirical data on the value of the parameter $\lambda^{2} / \gamma$ can be inferred from the returns of pension funds as reported for instance in OECD $(2018 a, 2018 b)$ and Better Finance (2019). Assumptions (7) lead to the value $1.65 \%$ for the expected excess return $\lambda^{2} / \gamma$.

${ }^{11}$ It may be noted that the assumption that the infinite-horizon scheme can borrow against future pension contributions becomes less credible when the interest rate is low, since under those circumstances a larger part of initial capital would come from contributions very far in the future.

${ }^{12}$ Within a general equilibrium setting, the equilibrium price of risk $\lambda$ can be related to more fundamental (but more difficult to measure) quantities such as the volatility of aggregate wealth and the risk aversion of a representative agent. For a fixed value of the volatility of aggregate wealth, a low value of $\lambda^{2} / \gamma$ should therefore be viewed as indicating a high level of risk aversion of the pension fund relative to the level of risk aversion of investors in general.
} 


\subsubsection{Deferred infinite-horizon scheme}

Under the infinite-horizon scheme, the generations that need to be compensated most are the ones that are close to retirement at the time at which the scheme is implemented. The investment horizon for these generations under the new scheme is short, and as a consequence (following the rule that welfare is measured in terms of expected utility of the benefit paid by the scheme, as discussed at the end of Section 2.2) they profit relatively little from the early-investment advantage expressed by the relation (10). Because the generations close to retirement form a burden for the infinite-horizon scheme, one might consider excluding these generations from participation in the new system. In other words, instead of implementing the infinite-horizon scheme at time 0 for all generations that are not yet retired, one might only implement it for generations that are more than $k$ years away from retirement, where $k$ is a constant to be chosen. One then has what might be called a deferred infinitehorizon scheme.

In the deferred scheme, the capital required at time 0 to realize a given certainty equivalent $C E$ for all participating generations is equal to

$$
\sum_{j=k+1}^{\infty} e^{-\left(r+\frac{1}{2} \lambda^{2} / \gamma\right) T_{j}} C E=\left(1+r_{d}\right)^{-k} \frac{1}{r_{d}} C E .
$$

The capital that is available from the contributions of the participating generations is equal to

$$
\sum_{j=k}^{\infty} e^{-r T_{j}} C=\left(1+r_{f}\right)^{-k} \frac{1}{r_{f}} C .
$$

Setting required capital equal to available capital, one finds that the certainty equivalent reached in the deferred infinite-horizon scheme with delay $k$ is equal to

$$
C E_{\mathrm{ih}, k}=\left(\frac{1+r_{d}}{1+r_{f}}\right)^{k} \frac{r_{d}}{r_{f}} C .
$$

This quantity is increasing in the length of the delay period. From (25) and (18) it follows that the deferred infinite-horizon scheme will be preferred by all generations to a moving-window scheme with window length $\tau$ if

$$
k>\tau-\tau_{\text {crit }}
$$

where $\tau_{\text {crit }}$ is given by (23). This gives another interpretation to the results in Table 1 . For instance, if the real interest rate is $2 \%$, the expected excess return $\lambda^{2} / \gamma$ is $3 \%$, and the window length is 45 years, then the table indicates that, to make the introduction of an infinite-horizon scheme acceptable at time 0 to all participating generations, one should exclude the generations that will retire in 7 years or less.

\subsection{The participation constraint}

Both in the infinite-horizon scheme and in moving-window schemes with sufficiently long time horizon, investments are already made on behalf of some of the participating generations before these generations enter the labor market, or even before they are born. Depending on the results of the investments, those generations may or may not be happy with the choices that have been made for them. In a stylized model, one can assume that there is a certain point in time at which generations can decide either to stay within the existing scheme or to invest for themselves. For instance, this point may be at the start of their working career, say at $T-\tau_{c}$ where $T$ is the time of retirement and $\tau_{c}$ 
denotes career length. When an incoming generation chooses not to participate in the existing scheme, it is said that discontinuation occurs. ${ }^{13}$

\subsubsection{Infinite-horizon scheme}

First, consider the case of the infinite-horizon scheme. If $\tau_{c}>\tau_{\text {crit }}$, then it follows from the discussion above that discontinuation already takes place at time 0 ; therefore, it will be assumed that

$$
\tau_{\text {ex }}:=\tau_{\text {crit }}-\tau_{c} \geq 0
$$

The parameter $\tau_{\text {ex }}$ will be referred to as the excess window length. At time 1, a participation decision will be taken by the generation retiring at time $\tau_{c}+1$, and so on. From the formula (14) for the benefit paid to the generation retiring at time $T$ under the infinite-horizon scheme and the formula (8) for the certainty equivalent of a benefit of the form $\alpha G_{T}^{1 / \gamma}$, it follows that the certainty equivalent of the benefit from the infinite-horizon scheme at time $T=t+\tau_{c}$, as seen from time $t$, is equal to

$$
C E_{T \mid t}^{\mathrm{ih}}=\left(G_{t} / G_{0}\right)^{1 / \gamma} \exp \left(-\frac{1}{\gamma}\left(r+\frac{1}{2} \lambda^{2} / \gamma\right) t\right) \frac{r_{d}}{r_{f}} C .
$$

This expression should be compared to the certainty equivalent that can be achieved by a generation that invests for itself during a period of length $\tau_{c}$, on the basis of the same contribution. By (18), this certainty equivalent is equal to $\exp \left(\left(\frac{1}{2} \lambda^{2} / \gamma\right) \tau_{c}\right) C$. Taking into account that $r_{d} / r_{f}=\exp \left(\left(\frac{1}{2} \lambda^{2} / \gamma\right) \tau_{\text {crit }}\right)$, the continuation condition may therefore be written as

$$
\left(G_{t} / G_{0}\right)^{1 / \gamma} \exp \left(-\frac{1}{\gamma}\left(r+\frac{1}{2} \lambda^{2} / \gamma\right) t\right) \geq \exp \left(-\left(\frac{1}{2} \lambda^{2} / \gamma\right) \tau_{\text {ex }}\right) .
$$

Using (4), the condition can be expressed in terms of the driving Brownian motion $W_{t}$, as follows:

$$
\frac{1}{2} \frac{\gamma-1}{\gamma} \lambda t+W_{t} \geq-\frac{1}{2} \lambda \tau_{\mathrm{ex}}
$$

The probability that discontinuation never occurs (as is assumed in the construction of the infinitehorizon scheme) is therefore equal to the probability that the above inequality is satisfied for all $t=1$, $2, \ldots$. As an approximation, one can use the probability that the inequality is satisfied for all $t \geq 0$ (i.e., including non-integer values). It is well known (see for instance Borodin and Salminen (2002, Part II, Section 2, formula 1.1.4(1))) that the probability of Brownian motion with positive drift, $W_{t}+a t$ with $a>0$, to reach a negative level $-b$ at any $t \geq 0$ is equal to $e^{-2 a b}$. If the drift is zero or negative, which happens in the situation here if the coefficient of risk aversion is smaller than or equal to 1 , then the level $-b$ is reached with probability 1 . From this, the discontinuation probability (in continuous approximation) is given, for $\gamma \geq 1$, by

$$
p_{\mathrm{disc}}^{\mathrm{ih}}=\exp \left[-\frac{1}{2} \frac{\gamma-1}{\gamma} \lambda^{2} \tau_{\mathrm{ex}}\right] .
$$

Under the parameter values stated in (7), the formula implies that the excess $\tau_{\text {ex }}$ of critical window length over the generations' window length that is needed to achieve a $5 \%$ discontinuation probability is approximately 91 years. The left panel of Figure 3 shows a plot of the probabilities of discontinuation on different horizons under the discrete version of the continuation condition (computed by

\footnotetext{
${ }^{13}$ In the literature, the participation constraint has been analyzed under different assumptions concerning the alternatives from which participants are able to choose, and the time points at which they can make a decision; see for instance Bovenberg et al. (2007), Beetsma et al. (2012), and Chen et al. (2017). Here, a relatively simple case is considered.
} 

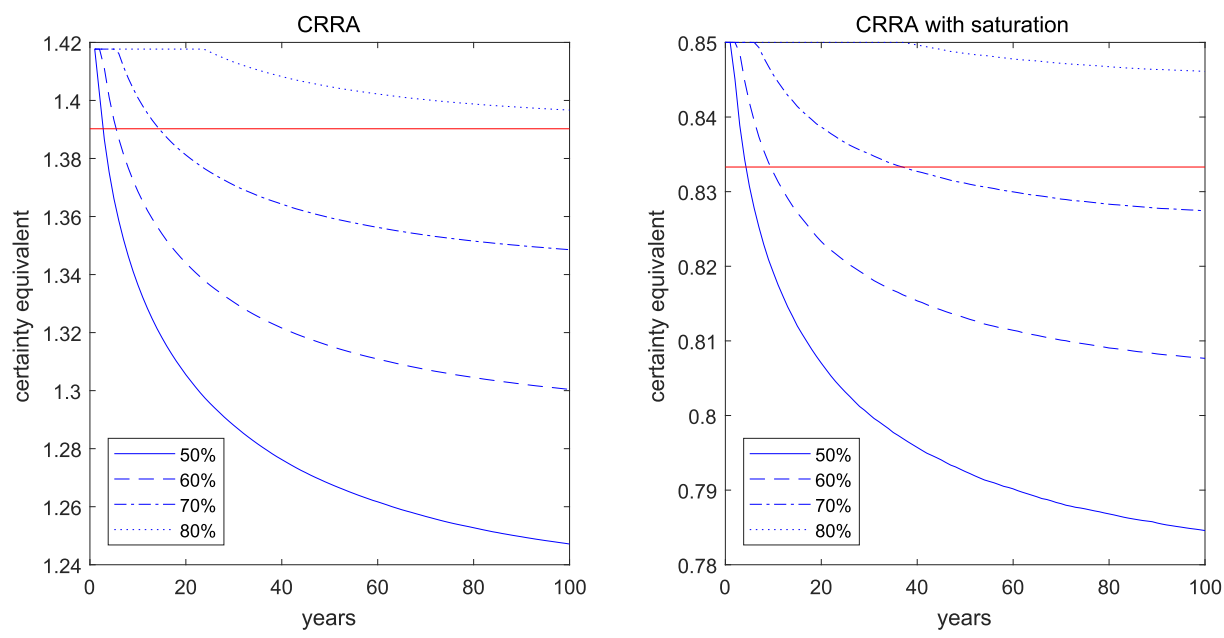

Figure 2. The figures show several quantiles (as listed in the legend) of the distribution of the minimum up to time $T$ of the certainty equivalent from the infinite-horizon scheme as experienced by incoming generations, as a function of $T$. Parameter values are as in (7). Standard CRRA utility is used in the left panel, CRRA with saturation (see Section 4.1) in the right panel. The contribution level in the left panel is 1 ; in the right panel, the contribution level is set such that the certainty equivalent for all generations as seen from the time of implementation of the scheme is equal to 0.85 . Also shown is a horizontal line indicating the level of certainty equivalent that generations would be able to achieve, on the basis of the same contribution level, by investing for themselves during a period of 40 years.
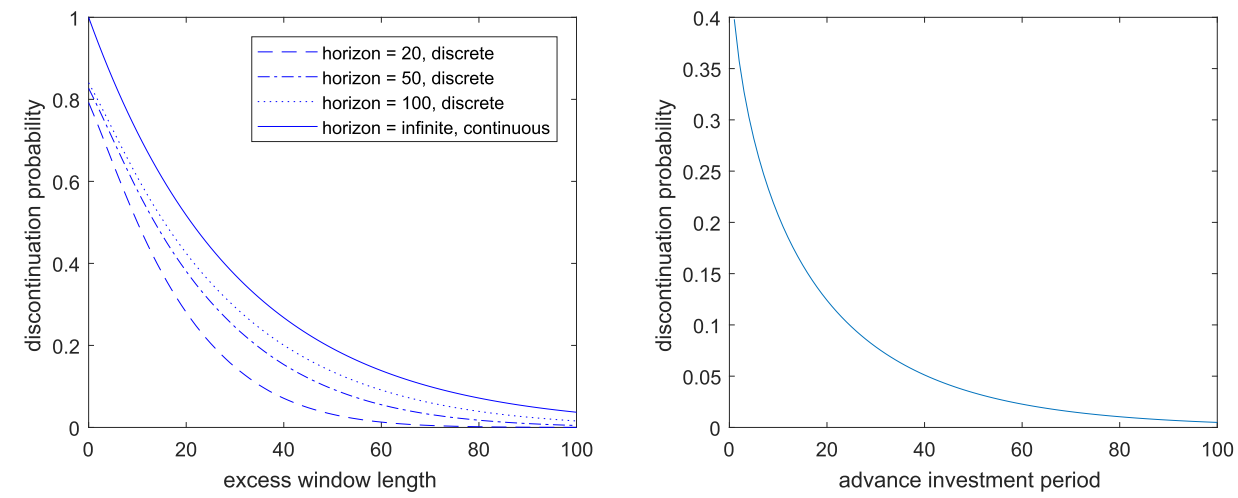

Figure 3. The left panel shows the probability of discontinuation for the infinite-horizon scheme during the first 20,50, or 100 years of existence of the scheme, as a function of excess window length. Also shown is the discontinuation probability for the entire (infinite) lifetime of the scheme, using a continuous approximation. The probabilities are given as a function of window length in excess of the critical value at which generations would already at time 0 decide in favor of a moving-window scheme. In the right panel, the discontinuation probability is shown for a single participating generation in the moving-window scheme, as a function of the length of the advance investment period. Parameter values are as in (7).

Monte Carlo), as well as a plot of the probability of discontinuation on the infinite horizon in the continuous version, for which the explicit formula (31) is available. All probabilities are expressed as functions of the excess window length.

The left panel of Figure 2 illustrates how discontinuation probabilities evolve as time progresses when the critical window length is computed from the parameter values in (7), and the comparison window length is 40 years. In this case, the excess window length is 2.37 years. It is seen that already after a few years, there is a $50 \%$ probability that at least one of the incoming generations would prefer to invest for itself during the proposed period of 40 years, rather than to join the infinite-horizon scheme. 


\subsubsection{Moving-window scheme}

Consider now the participation constraint in the moving-window scheme. Let $\tau_{c}$ denote, as above, the investment window length that is available to generations by themselves, and assume that the window length used by the moving-window scheme satisfies $\tau>\tau_{c}$. Define the advance investment period $\tau_{a}$ by

$$
\tau_{a}=\tau-\tau_{c}>0
$$

Suppose that the generation that will retire at time $t+\tau_{c}$ is allowed to choose at time $t$ between either participating in a fixed-window scheme with window length $\tau$, which already has been started up at time $t-\tau_{a}$, or investing for itself during the period from $t$ to $T=t+\tau_{c}$. At time $t$, preliminary investment results of the moving-window scheme that has started up at time $t-\tau_{a}$ are already known, and the generation retiring at time $T$ is able to let its decision depend on those investment results. It follows from the expression (19) for the benefit received at time $T$ under the moving-window scheme and the formula (8) for the certainty equivalent of a payoff of the form $\alpha G_{T}^{1 / \gamma}$ that the certainty equivalent of the benefit (19) received at time $T$, as seen from time $t$, is given by

$$
C E_{T \mid t}^{\mathrm{mw}}=\left(\frac{G_{t}}{G_{t-\tau_{a}}}\right)^{1 / \gamma} \exp \left[\frac{1}{\gamma}\left(r+\frac{1}{2} \frac{\lambda^{2}}{\gamma}\right) \tau_{c}-\frac{1}{\gamma}\left(r-\frac{1}{2} \frac{\gamma-1}{\gamma} \lambda^{2}\right) \tau\right] C .
$$

This should be compared to the certainty equivalent of investing across a period of length $\tau_{c}$, which is equal to $\exp \left(\frac{1}{2}\left(\lambda^{2} / \gamma\right) \tau_{c}\right) C$. The continuation condition can therefore be written as

$$
\frac{1}{\tau_{a}} \log \frac{G_{t}}{G_{t-\tau_{a}}}-r>-\frac{1}{2} \frac{\gamma-1}{\gamma} \lambda^{2} .
$$

In other words, the generation will participate in the moving-window scheme if the annualized excess geometric return of the optimal growth portfolio during the interval from $t-\tau_{a}$ to $t$ is larger than a certain lower bound, which under the assumptions of (7) is $-3.3 \%$. The discontinuation probability for a scheme with advance investment period $\tau_{a}$ is given by

$$
p_{\mathrm{disc}}^{\mathrm{mw}}=\Phi\left(-\frac{\gamma-\frac{1}{2}}{\gamma} \lambda \sqrt{\tau_{a}}\right)
$$

A plot of the discontinuation probability as a function of the advance investment period is shown in the right panel of Figure 3.

\section{Alternative preference specifications}

It may be argued that the objective of a second-pillar pension plan is to offer participants the means to uphold their standard of living after retirement. To express this objective, the standard CRRA utility function has certain drawbacks. First, expected utility under CRRA is sensitive to the possible occurrence of high returns. From a second-pillar perspective, such returns are less interesting when they bring more than what is needed to maintain the standard of living of participants. Participants who are interested in speculative opportunities can use a private (third-pillar) pension plan for this goal. At the same time, CRRA utility may not sufficiently punish scenarios in which returns are low and which would provide participants with income beneath what might be called a subsistence level. Similar arguments pleading for minimum (subsistence) and maximum (saturation) constraints in the context of pension funds have been brought forward for instance by Martellini and Milhau (2012).

To express the concerns mentioned above, two fairly simple modifications of CRRA utility will be employed in this section. First, a saturation level is brought in; this reduces the speculative element in CRRA without letting the coefficient of risk aversion tend to infinity, which would suppress risk taking 
entirely. In a second modification, a guaranteed minimum income after retirement is introduced. It is possible to introduce both saturation and subsistence by means of smooth utility functions, as shown by Conniffe (2007). However, simpler expressions for the optimal payoffs are obtained when kinked utility functions are used, as for instance in Yamashita (2014), and this is the road that will be followed here.

\subsection{Adding a saturation level}

The modified CRRA utility function that will be considered in this subsection is given by

$$
u(x)=\min \left(\frac{x^{1-\gamma}}{1-\gamma}, \frac{1}{1-\gamma}\right),
$$

where, without loss of generality, the level at which saturation takes place is set equal to 1 . It is readily verified that, under this preference specification, the highest utility for a given level of available capital is achieved by a payoff of the form

$$
B_{T}=\min \left(\left(G_{T} / K\right)^{1 / \gamma}, 1\right)
$$

where $K>0$ is a parameter. The expression (36) can be read as the payoff formula of what might be called a 'capped power option', with $G_{T}$ playing the role of the value of the underlying at expiry, and $K$ acting as a strike level. For values of $G_{T}$ less than $K$, the payoff is given by $\left(G_{T} / K\right)^{1 / \gamma}$, whereas the payoff is equal to the constant 1 when $G_{T}>K$. The certainty equivalent at time $T$ of the payoff min $\left(\left(G_{T} / K\right)^{1 / \gamma}, 1\right)$ and the capital required at time 0 to generate this payoff are given, respectively, by (see Appendix)

$$
\begin{aligned}
& C E(K, T)=\left[\frac{\varphi\left(d_{1}\right)}{\varphi\left(d_{\gamma}\right)} \Phi\left(-d_{\gamma}\right)+\Phi\left(d_{1}\right)\right]^{1 /(1-\gamma)} \\
& v_{0}(K, T)=e^{-r T}\left[\frac{\varphi\left(d_{2}\right)}{\varphi\left(d_{\gamma}\right)} \Phi\left(-d_{\gamma}\right)+\Phi\left(d_{2}\right)\right],
\end{aligned}
$$

where ${ }^{14}$

$$
\begin{gathered}
d_{1}=\frac{\log \left(G_{0} / K\right)+\left(r+\frac{1}{2} \lambda^{2}\right) T}{\lambda \sqrt{T}}, \quad d_{2}=d_{1}-\lambda \sqrt{T}, \\
d_{\gamma}=d_{1}-\frac{\gamma-1}{\gamma} \lambda \sqrt{T}=d_{2}+\frac{1}{\gamma} \lambda \sqrt{T} .
\end{gathered}
$$

The notation $d_{1}\left(K, T, G_{0}\right)$, etc., will also be used, to stress that the parameters $d_{1}, d_{2}$, and $d_{\gamma}$ are functions of the strike level $K$, the payment date $T$, and the initial value $G_{0}$ of the growth optimal portfolio. The expressions (37), (38), and (39) take the place of relations (8) and (9) in the CRRA case.

Since both the certainty equivalent at time $T$ and the time- 0 value are strictly decreasing functions of the strike $K$, there is, for fixed $T$, a one-one relation between them. The certainty equivalent can for instance be looked at as a function of the time- 0 value. In contrast to the CRRA case, the relationship is not linear. Due to the saturation, the certainty equivalent does not increase anymore when the

\footnotetext{
${ }^{14}$ The symbol $d_{2}$ is used here in line with tradition in mathematical finance, although it should actually be named $d_{\infty}$ to be consistent with the definition of $d_{\gamma}$.
} 

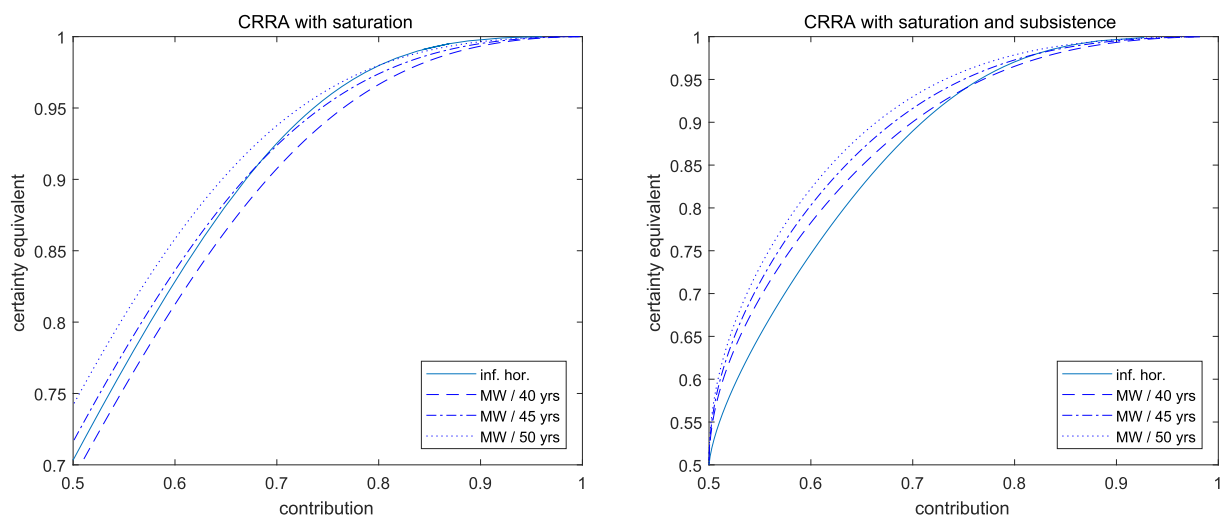

Figure 4. Both panels show the dependence of a certainty equivalent of the optimal benefit for the infinite-horizon scheme as well as for the moving-window scheme with different window lengths. In the left panel, a CRRA utility function is used with saturation at level 1. The right panel adds a subsistence level at $\eta=0.5$ (see Section 4.2). Specifications of the corresponding optimal benefits are given in (36) and in (43), respectively. Parameter values are as in (7).

time- 0 value is raised above the level $e^{-r T}$, which is the amount from which the saturation level 1 can be generated by investing only in riskless assets. Consequently, in any scheme in which the contribution paid by generations, lumped to time $T$, is equal to the financially fair value $e^{r T} v_{0}(T)$, the amount of the contribution should not exceed the saturation level 1 . Plots of the certainty equivalent as a function of the financially fair contribution are shown in Figure 4.

The ratio of certainty equivalent to a financially fair contribution can also be viewed as a measure of the extent to which investing in risky assets is better (on an ex-ante basis) than using only a riskless account. Under CRRA preferences, this ratio is constant as a function of the contribution level, and is exponentially increasing as a function of the investment horizon. This supports the commonly held opinion that risky investments are relatively more attractive on longer time scales; it also motivates pension schemes that aim to provide participants with longer investment horizons than they would be able to realize for themselves, such as the infinite-horizon scheme and the moving-window schemes with large window length. The left panel of Figure 5 shows the ratio of certainty equivalent to contribution level as a function of the contribution level for several values of the investment horizon. It is seen that the positive effect of horizon lengthening is not affected for low values of the contribution level, but is substantially reduced for higher levels. The same phenomenon is shown in a different way in the right panel of Figure 5, which shows a plot of the improvement of the certainty equivalent obtained by lengthening the horizon, relative to the level of certainty equivalent, as a function of horizon length for several values of the contribution level. ${ }^{15}$ Under the CRRA preferences, this relative improvement function is a constant (namely $\frac{1}{2} \lambda^{2} / \gamma$ ). It is seen that, when saturation is added, the relative improvement of the certainty equivalent goes down considerably with increasing horizon length, especially when the contribution level is close to the saturation level.

Under the infinite-horizon scheme, the contribution lumped to the time of retirement (the same for all generations) that corresponds to a given level of the time- $T$ certainty equivalent $C E$ (also the same for all generations) can be computed as follows. First, determine the strike levels $K_{j}(j=1,2$, ... ) from the equations

$$
\frac{\varphi\left(d_{1}^{j}\right)}{\varphi\left(d_{\gamma}^{j}\right)} \Phi\left(-d_{\gamma}^{j}\right)+\Phi\left(d_{1}^{j}\right)=C E^{1-\gamma}, \quad d_{1}^{j}:=d_{1}\left(K_{j}, T_{j}, G_{0}\right), \quad d_{\gamma}^{j}:=d_{\gamma}\left(K_{j}, T_{j}, G_{0}\right) .
$$

\footnotetext{
${ }^{15}$ In other words, the plot shows $(\partial C E(T, c) / \partial T) / C E(T, c)$ as a function of $T$ for several values of the financially fair contribution level $c$.
} 

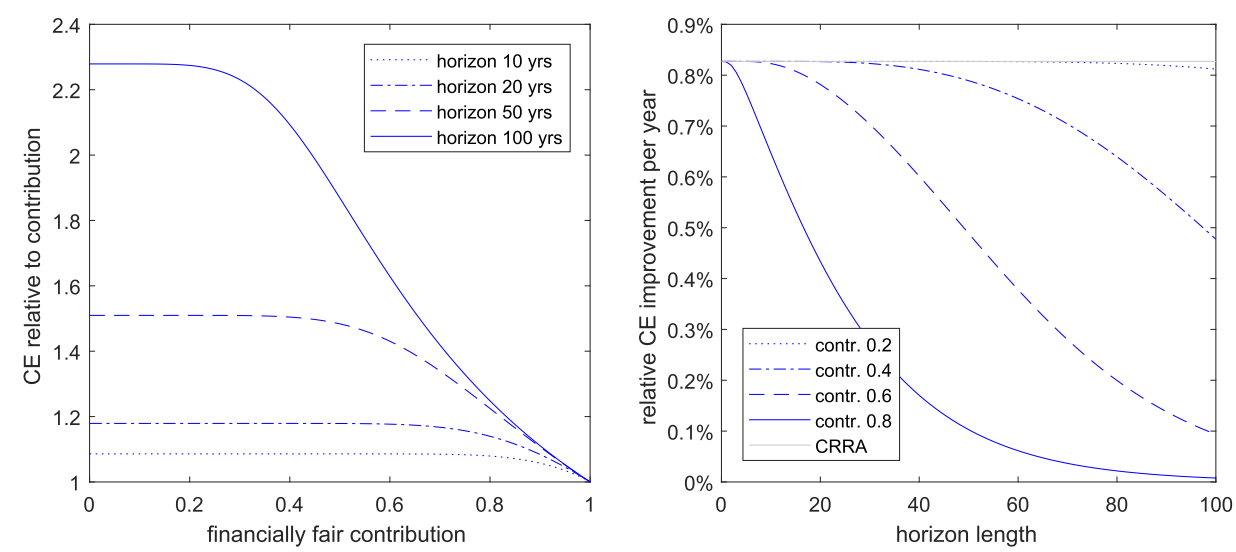

Figure 5. The two panels show, in different ways, the improvement that is obtained in the relation between certainty equivalent and required capital when the investment horizon is increased, assuming a saturated CRRA utility function as specified in (35). Parameter values are as in (7). The left panel shows time- $T$ certainty equivalent relative to financially fair contribution lumped to time $T$ as a function of the contribution level, for several values of the investment horizon. The right panel shows the relative increase of certainty equivalent for each year of horizon lengthening, for several values of the contribution level.

Then, determine the corresponding contribution level from $C=r_{f} A_{0}$ where

$$
A_{0}=\sum_{j=1}^{\infty} v_{0}^{j}, \quad v_{0}^{j}=e^{-r T_{j}}\left[\frac{\varphi\left(d_{2}^{j}\right)}{\varphi\left(d_{\gamma}^{j}\right)} \Phi\left(-d_{\gamma}^{j}\right)+\Phi\left(d_{2}^{j}\right)\right], \quad d_{2}^{j}:=d_{2}\left(K_{j}, T_{j}, G_{0}\right) .
$$

It is readily verified that this establishes a strictly increasing relationship between the certainty equivalent $C E$ and the contribution level $C$. The benefit paid at time $T_{j}$ in the infinite-horizon scheme is given by (36) with $T=T_{j}$ and $K=K_{j}$ as determined by (40). The benefit may be considered as a function of $C E$, but due to the monotonic relationship between $C E$ and $C$, it can also be considered as a function of the contribution level.

Remark 4.1. If the initial value $G_{0}$ of the growth optimal portfolio is replaced by another value $\tilde{G}_{0}$, then it is seen from definitions (39) that the solutions of (40) will be given by $\tilde{K}_{j}=a K_{j}$, where $a=\tilde{G}_{0} / G_{0}$. Again from (39) it then follows that the time- 0 values $v_{0}^{j}$ are not affected, so that also the contribution level $C$ remains the same. Therefore, in computations to determine the relationship between contribution and certainty equivalent, one can set $G_{0}$ to any positive value.

Within the moving-window scheme with window length $\tau$, the contribution level that corresponds to a given certainty equivalent can be found by first solving for $K$ from the relationships (37) and (39), with $T$ replaced by $\tau$, and subsequently computing $C=e^{r \tau} v_{0}(\tau)$ where $v_{0}(\tau)$ is given by (38). Remark 4.1 also applies in this case. When the strike $K$ is computed using $G_{0}=1$, then the benefit at time $T_{j}$ under the moving-window scheme is given by (36) with $G_{T}$ replaced by $G_{T_{j}} / G_{T_{j}-\tau}$.

On the basis of the relations that are thus established between certainty equivalent and contribution level both in the infinite-horizon scheme and in the moving-window scheme, one can now compare the certainty equivalents that can be reached on the basis of a given contribution level within these schemes. In contrast to the CRRA case, the comparison depends on the contribution level. Figure 4 shows a graph of the certainty equivalent of benefits as a function of the contribution level, for the infinite-horizon scheme as well as for three moving-window schemes with different window lengths. In all schemes, the contribution level can be reduced substantially from the maximal level 1 with only a moderate decrease of the certainty equivalent; this shows the positive impact of investing in risky assets at long time horizons. At low levels of the contribution, the relative 
performance of schemes is the same as in the CRRA case. However, at contribution levels closer to the saturation level, the infinite-horizon scheme climbs up higher in the hierarchy, surpassing the 50 -year moving-window scheme at approximately the 0.8 level. It may therefore be concluded that the infinite-horizon scheme profits more (or suffers less) from the effects of saturation than the moving-window schemes do.

The impact of the participation constraint is illustrated in the right panel of Figure 2. The situation is considered in which the contribution level is chosen in such a way that the certainty equivalent 0.85 , as seen from time 0 , is realized for all generations in the infinite-horizon scheme, under assumptions that are the same as the ones that apply to Figure 4. Computation shows that the same contribution level in the moving-window scheme with a window length of 40 years leads to the value 0.8327 for the certainty equivalent. For any incoming generation, this number can be compared to the certainty equivalent of the benefit from the infinite-horizon scheme, conditional upon the level of the growth optimal portfolio at the time at which the generation takes its participation decision. For a generation that decides in year $j$ and retires in year $j+n$, the conditional certainty equivalent is determined by the formulas (37) and (39) in which $G_{0}$ is replaced by $G_{T_{j}}, K=K_{j+n}$ as determined in (40), and $T=n$. These formulas do not allow for analytic calculation of the probability that the conditional certainty equivalent from the infinite-horizon scheme will cross the certainty equivalent from the moving-window scheme before a given time, but the calculation of these probabilities by Monte Carlo simulation is straightforward. The resulting curves are shown in the right panel of Figure 2. It is seen that the situation has improved somewhat with respect to the CRRA case; nevertheless, in more than $50 \%$ of the scenarios, there is still within 10 years an incoming generation that, on the basis of information available when it starts its working career, would choose not to participate in the infinite-horizon scheme. The probability increases to $70 \%$ after 50 years.

\subsection{Adding a subsistence level}

As a further modification of the standard CRRA utility function, consider now a situation in which, in addition to the saturation level normalized to 1 , a subsistence level is introduced which represents the minimum benefit that is required by agents. The subsistence level will be denoted by $\eta<1$. Accordingly, the utility function is now defined as follows:

$$
u(x)=\left\{\begin{array}{cl}
1 & \text { if } x \geq 1 \\
\frac{x^{1-\gamma}}{1-\gamma} & \text { if } \eta \leq x<1 \\
-\infty & \text { if } x<\eta
\end{array}\right.
$$

By assigning utility value $-\infty$ to payments less than $\eta$, it is ensured that optimal solutions will always pay at least $\eta$, so that effectively the level $\eta$ is given the role of a guaranteed minimum. In this case, the optimal contingent benefit is of the form

$$
B_{T}=\left\{\begin{array}{cl}
1 & \text { if } G_{T} \geq K \\
\left(G_{T} / K\right)^{1 / \gamma} & \text { if } \bar{K} \leq G_{T} \leq K \\
\eta & \text { if } G_{T} \leq \bar{K}
\end{array}\right.
$$

where $\bar{K}$ is defined by

$$
\bar{K}=\eta^{\gamma} K
$$

As before, the parameter $K$ can be used as a mediating variable to strike a compromise between contribution on the one hand and the certainty equivalent of benefits on the other. Both are strictly increasing functions of $K$. One can compute (see Appendix) that the certainty equivalent of the benefit 
defined in (43) and the time- 0 value of this benefit are given, respectively, by

$$
\begin{gathered}
C E=\left[\eta^{1-\gamma} \Phi\left(-\bar{d}_{1}\right)+\frac{\varphi\left(d_{1}\right)}{\varphi\left(d_{\gamma}\right)}\left(\Phi\left(\bar{d}_{\gamma}\right)-\Phi\left(d_{\gamma}\right)\right)+\Phi\left(d_{1}\right)\right]^{1 /(1-\gamma)} \\
v_{0}=e^{-r T}\left[\eta \Phi\left(-\bar{d}_{2}\right)+\frac{\varphi\left(d_{2}\right)}{\varphi\left(d_{\gamma}\right)}\left(\Phi\left(\bar{d}_{\gamma}\right)-\Phi\left(d_{\gamma}\right)\right)+\Phi\left(d_{2}\right)\right]
\end{gathered}
$$

where $d_{1}, d_{2}$, and $d_{\gamma}$ are as defined in (39), and $\bar{d}_{1}, \bar{d}_{2}$, and $\bar{d}_{\gamma}$ are the analogous quantities with $K$ replaced by $\bar{K}$ :

$$
\begin{gathered}
\bar{d}_{1}=\frac{\log \left(G_{0} / \bar{K}\right)+\left(r+\frac{1}{2} \lambda^{2}\right) T}{\lambda \sqrt{T}}, \quad \bar{d}_{2}=\bar{d}_{1}-\lambda \sqrt{T}, \\
\bar{d}_{\gamma}=\bar{d}_{1}-\frac{\gamma-1}{\gamma} \lambda \sqrt{T}=\bar{d}_{2}+\frac{1}{\gamma} \lambda \sqrt{T} .
\end{gathered}
$$

The contribution needs to be at least equal to $\eta$; otherwise, it is not possible to guarantee a minimum benefit at this level. Setting the contribution equal to $\eta$ in fact comes down to selecting a deterministic pension savings scheme; the certainty equivalent is in that case equal to $\eta$ as well.

The relation between contributions and certainty equivalents can be computed analogously to the case of the previous section. The right panel of Figure 4 shows the effect of the addition of a minimum guarantee at $50 \%$ of the saturation level on the comparison between the infinite-horizon scheme and moving-window schemes with several window lengths. As in the case with only saturation, the certainty equivalent goes down initially very slowly as the contribution is reduced from its maximal value. The upward effect on the infinite-horizon scheme, compared to the CRRA case, is still visible for contribution levels that are close to the saturation level. However, at lower contribution levels, the performance of the infinite-horizon scheme weakens considerably relative to the moving-window schemes.

\section{Conclusions}

This paper has revisited the model for collective pension schemes that has been proposed by Gollier (2008). While the main stylized structure of the model has not been changed, an important modification concerns the treatment of generations that are between career start and retirement at the time at which a transition to an infinite-horizon scheme takes place. The present paper has investigated the situation in which the net investment gains (or losses) experienced by these generations are left in their hands, contrary to the assumptions of Gollier (2008). Moreover, a second-pillar point of view has been used to motivate modifications to the standard CRRA utility function.

In the Black-Scholes economy under the classical CRRA preferences, the level of utility of optimally invested capital, as measured in terms of certainty equivalents, increases more quickly than the rate of interest on capital. In other words, less money is needed to reach a certain welfare level when an investment is started earlier, even taking into account the interest rate. The concept of 'horizon lengthening' is an important motivation of collective pension schemes. The present paper is, like Gollier (2008), a study in the implementation of this concept. A distinction has been made in this paper between infinite-horizon schemes and moving-window schemes (with different lengths of the investment window), which does not quite coincide with the distinction between 'collective' and 'autarky' in Gollier (2008). 
A first result from computations in this paper concerns the impact of assumptions concerning the treatment of net returns on investments achieved by generations that are working at the time of the transform. The position taken in this paper is that these net returns remain in the hands of these generations, whereas it is assumed in Gollier (2008) that they will be absorbed into the capital of the collective system, and moreover that they will take their expected value. Under the parameter assumptions of Gollier (2008), it has been shown in this paper that there is a $14.6 \%$ reduction of initial capital available to the infinite-horizon scheme when the first position is taken rather than the second. This covers a significant part of the $19 \%$ increase in pension benefits that is realized by means of the infinite-horizon scheme as reported in Gollier (2008, p. 1473).

Under the modified assumption concerning the ownership rights of the net investment results of working generations, replacement of an existing moving-window scheme by an infinite-horizon scheme is not always recommended. In the BS/CRRA model, the decision depends on (i) the interest rate, (ii) the parameter $\lambda^{2} / \gamma$, which represents the expected annual arithmetic excess return of a CRRA investor with risk aversion parameter $\gamma$ in a BS economy with the price of risk given by $\lambda$, and (iii) the length of the investment window in the moving-window scheme. For the infinite-horizon scheme to be preferred, the 'critical window length' that can be computed from the interest rate and the parameter $\lambda^{2} / \gamma$ should be larger than the window length of the existing moving-window scheme. If it is assumed that the latter window length is equal to career length, say 40 or 45 years, then, as shown in Table 1, under realistic parameter values the decision may go either way. The infinite-horizon scheme is in particular preferred in situations in which both the interest rate and the expected excess return $\lambda^{2} / \gamma$ are fairly low.

The reason why the infinite-horizon scheme is not always preferred is that a comparison is made on the basis of expected utility from the benefit provided by the scheme, and generations that participate in the scheme and that are already close to retirement are confronted with a rather short investment horizon. The balance of preference can be shifted towards the infinite-horizon scheme by excluding these generations when the new scheme is introduced; the critical window length increases by the number of generations that are skipped.

To make the preference for the infinite-horizon scheme robust against future market developments, so that one can be reasonably sure that future generations will continue to participate (as is assumed in the infinite-horizon scheme), it turns out that the critical window length must in fact be quite a bit larger than the length of the investment window that individuals would be able to realize for themselves. The left panel of Figure 3 indicates that the excess window length (i.e., the difference between the critical window length and the length of the investment window in a competing moving-window scheme) should be at least 60 to arrive at a value of the discontinuation probability close to $5 \%$. The right panel of the same figure shows that, in a moving-window scheme, a comparable level of robustness against discontinuation risk can only be achieved by starting investments on behalf of a given generation already 40 years before this generation enters the labor market.

Under modifications of the CRRA utility function that are proposed here to express more strongly the perspective of second-pillar pension provision, the claim that horizon lengthening increases welfare remains true, although the effect is weaker than in the classical CRRA case; this is illustrated in Figures 5 and 6 . The weakening of the effect of horizon lengthening is due to the fact that both saturation and the introduction of a subsistence level call for portfolio derisking under some circumstances, so that the benefit of investing in risky assets is felt less. Unlike the classical CRRA case, preference for either an infinite-horizon or a moving-window scheme becomes dependent on the amount of contribution that generations are willing to pay to save for retirement. The two panels of Figure 4 show that the infinite-horizon scheme does relatively well (with respect to the CRRA case) for high contribution levels, but becomes less attractive at lower contribution levels, especially in the case in which both a saturation level and a subsistence level are present.

The relative comparison of the infinite-horizon scheme and moving-window schemes becomes increasingly less realistic when lower values for the real interest rate are assumed, as already mentioned in footnote 11. Under the assumption $r<0$, which means that the real interest rate is negative for all 

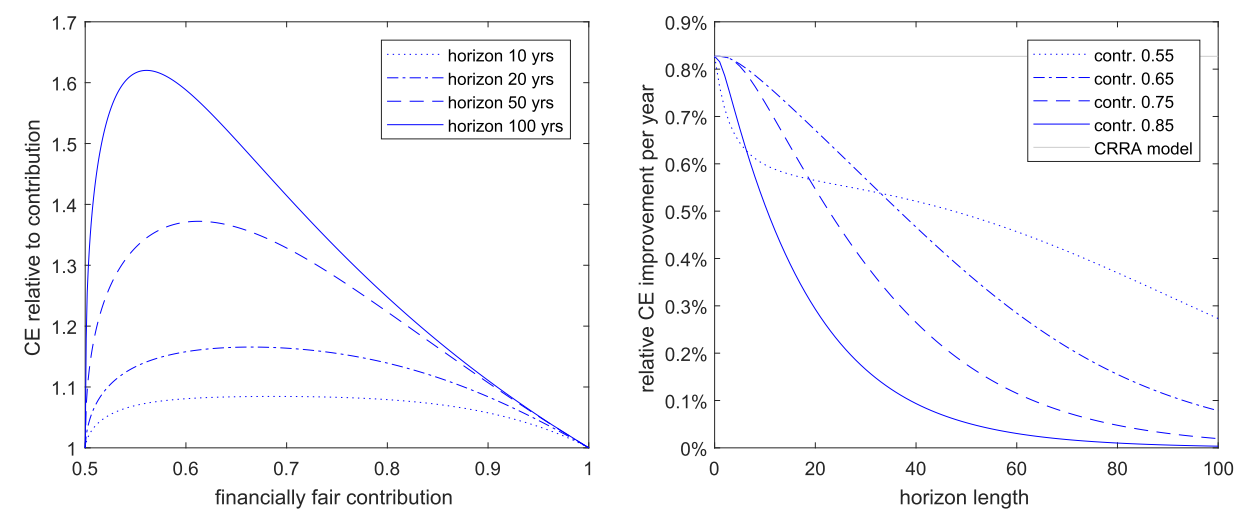

Figure 6. The figure is analogous to Figure 5, but now assuming a subsistence level $\eta=0.5$ as specified in (43). Since the contribution must be at least equal to $\eta$, the range of contribution levels is not the same as in Figure 5 .

maturities and for all time to come, the assumption of the infinite-horizon scheme that the net present value of all future contributions is available at time 0 even becomes completely meaningless, since it would imply that the amount of money that the collective fund has at its disposal at the time of initiation of the scheme is infinite. The results of the comparison at low interest rates should therefore be interpreted with care. It may be better under such circumstances to limit the use of the model to mutual comparison of moving-horizon schemes with investment horizons that are not excessively long.

The results that have been obtained in this paper, in particular concerning discontinuation risk, may lead policy makers to be skeptical about the feasibility both of the infinite-horizon scheme and of moving-window schemes with long investment windows. There is still a role for collective schemes, however, even when only moving-window schemes are considered with window lengths of the same magnitude as typical career lengths. The collective can overcome the credit constraints with which individuals are confronted, so that effectively they are still able to implement horizon lengthening to a certain extent. In other words, intergenerational risk sharing still has a positive effect.

In this note, some aspects that are discussed extensively in Gollier (2008) have been left out of consideration. This includes possible ways of mitigating discontinuation risk by means of risk sharing with outside parties. Another aspect that receives considerable attention in Gollier (2008) is the investment policy (risk taking) of collective pension funds. This aspect has not been discussed in the present paper, but the investment policies related to all schemes that have been under consideration here can be computed straightforwardly as the replicating portfolios for the corresponding payoffs.

The analysis of this paper rests on assumptions that are quite simple and that could be replaced by different and possibly more complicated assumptions. This refers both to the modeling of agents' preferences and to the description of the economic environment. Important topics for analysis that cannot be addressed within the context of the model discussed in this paper include the following: (i) the impact of the variability of interest rates; (ii) the role of the variability of contributions which may be due to wage fluctuations, which in turn may be related to capital market returns; (iii) demographic developments and the relation to intergenerational risk sharing in the first pillar. Nevertheless, the model constructed in Gollier (2008) is still useful to highlight the role played by basic economic parameters in tradeoffs that arise in the design of collective pension schemes in the second pillar.

Acknowledgements. I am grateful to two anonymous reviewers for their comments and suggestions.

\section{References}

Ball L and Mankiw NG (2007) Intergenerational risk sharing in the spirit of Arrow, Debreu, and Rawls, with applications to social security design. Journal of Political Economy 115, 523-547. 
Beetsma RMWJ and Romp WE (2016) Intergenerational risk sharing. In Piggott J and Woodland A (eds), Handbook of the Economics of Population Aging, vol. 1. North Holland, Amsterdam: Elsevier, pp 311-380.

Beetsma RMWJ, Romp WE and Vos SJ (2012) Voluntary participation and intergenerational risk sharing in a funded pension system. European Economic Review 56, 1310-1324.

Better Finance (Ján Šebo and Ştefan Dragoş Voicu, coordinators) (2019) Pension savings: the real return - 2018 edition. Available at ssrn.com/abstract $=3412215$.

Borodin AN and Salminen P (2002) Handbook of Brownian Motion - Facts and Formulae, 2nd Edn. Basel: Birkhäuser.

Bovenberg L and Mehlkopf R (2014) Optimal design of funded pension schemes. Annual Review of Economics 6, 445-474.

Bovenberg L, Koijen R, Nijman T and Teulings C (2007) Saving and investing over the life cycle and the role of collective pension funds. De Economist 155, 347-415.

Chen DHJ, Beetsma RMWJ, Ponds EHM and Romp WE (2016) Intergenerational risk-sharing through funded pensions and public debt. Journal of Pension Economics \& Finance 15, 127-159.

Chen DHJ, Beetsma RMWJ, Broeders DWGA and Pelsser AAJ (2017) Sustainability of participation in collective pension schemes: an option pricing approach. Insurance: Mathematics and Economics 74, 182-196.

Conniffe D (2007) The flexible three parameter utility function. Annals of Economics \& Finance 8, 57-63.

Cui J, de Jong F and Ponds E (2011) Intergenerational risk sharing within funded pension schemes. Journal of Pension Economics \& Finance 10, 1-29.

Gollier C (2008) Intergenerational risk-sharing and risk-taking of a pension fund. Journal of Public Economics 92, 14631485.

Gordon RH and Varian HR (1988) Intergenerational risk sharing. Journal of Public Economics 37, 185-202.

Krueger D and Kubler F (2006) Pareto-improving social security reform when financial markets are incomplete!? American Economic Review 96, 737-755.

Martellini L and Milhau V (2012) Dynamic allocation decisions in the presence of funding ratio constraints. Journal of Pension Economics \& Finance 11, 549-580.

Merton RC (1969) Lifetime portfolio selection under uncertainty: the continuous-time case. Review of Economics and Statistics 51, 247-257.

Merton RC (1990) Continuous-time Finance. Cambridge, MA: Blackwell.

OECD (2018a) Survey of large pension funds and public pension reserve funds, 2016. Available at www.oecd.org/daf/fin/private-pensions/2016-Large-Pension-Funds-Survey.pdf.

OECD (2018b) Pension markets in focus 2018. Available at http://www.oecd.org/daf/fin/private-pensions/Pension-Marketsin-Focus-2018.pdf.

Teulings CN and de Vries CG (2006) Generational accounting, solidarity and pension losses. De Economist 154, 63-83.

Yamashita M (2014) Optimal investment strategy for kinked utility maximization: covered call option strategy. Journal of Mathematical Finance 4, 55-74.

\section{Appendix A}

The expressions for time- 0 values and certainty equivalents given in Section 4.1 can be derived from the following formula, which holds for $\sigma>0,0<\eta \leq 1$, and $Z \sim N(0,1)$ :

$$
E[\max (\eta, \min (\exp (\sigma(Z+d)), 1))]=\eta \Phi(-\bar{d})+\frac{\varphi(d)}{\varphi(d+\sigma)}[\Phi(\bar{d}+\sigma)-\Phi(d+\sigma)]+\Phi(d)
$$

where

$$
\bar{d}=d-\frac{\log \eta}{\sigma}
$$

To derive (A.1), note that the equalities $\exp (\sigma(z+d))=\eta$ and $\exp (\sigma(z+d))=1$ hold for $z=-\bar{d}$ and $z=-d$, respectively. Write the expectation in (A.1) as $E_{1}+E_{2}+E_{3}$ where $E_{1}=\int_{-\infty}^{-\bar{d}} \eta d \Phi(z)=\eta \Phi(-\bar{d}), E_{3}=\int_{-d}^{\infty} 1 d \Phi(z)=\Phi(d)$, and finally

$$
\begin{aligned}
E_{2} & =\frac{1}{\sqrt{2 \pi}} \int_{-\bar{d}}^{-d} \exp (\sigma(z+d)) \exp \left(-\frac{1}{2} z^{2}\right) d z=\frac{\exp \left(\sigma d+\frac{1}{2} \sigma^{2}\right)}{\sqrt{2 \pi}} \int_{-\bar{d}}^{-d} \exp \left(-\frac{1}{2}(z-\sigma)^{2}\right) d z= \\
& =\frac{\varphi(d)}{\varphi(d+\sigma)}[\Phi(\bar{d}+\sigma)-\Phi(d+\sigma)] .
\end{aligned}
$$


This completes the derivation of (A.1). By taking the limit of both sides of (A.1) as $\eta$ tends to 0 , one finds the formula

$$
E[\min (\exp (\sigma(Z+d)), 1)]=\frac{\varphi(d)}{\varphi(d+\sigma)}[1-\Phi(d+\sigma)]+\Phi(d) .
$$

Of course, this can also be obtained by direct integration. Now, to find the time- 0 value of the payoff $B_{T}$ defined in (43), note that

$$
\log \left(\left(G_{T} / K\right)^{1 / \gamma}\right)=\frac{1}{\gamma}\left(\log \left(G_{0} / K\right)+\left(r-\frac{1}{2} \lambda^{2}\right) T+\lambda \sqrt{T} Z\right)=\frac{\lambda}{\gamma} \sqrt{T}\left(Z+d_{2}\right),
$$

where $Z$ is standard normal under $Q$, and where $d_{2}$ is defined in (39). Applying the formula (A.1), one finds (46). Furthermore, to compute the certainty equivalent under the preference specification (42) of the payoff $B_{T}$, write

$$
\log \left(G_{T} / K^{(1-\gamma) / \gamma}\right)=\frac{1-\gamma}{\gamma}\left(\log \left(G_{0} / K\right)+\left(r+\frac{1}{2} \lambda^{2}\right) T+\lambda \sqrt{T} Z\right)=\frac{1-\gamma}{\gamma} \lambda \sqrt{T}\left(Z+d_{1}\right)
$$

where, this time, $Z$ is standard normal under $P$, and $d_{1}$ is as defined in (39). The certainty equivalent satisfies the equality

$$
C E^{1-\gamma}=E\left[\max \left(\eta^{1-\gamma}, \min \left(\exp \left(\frac{1-\gamma}{\gamma} \lambda \sqrt{T}\left(Z+d_{1}\right)\right), 1\right)\right)\right]
$$

Applying (A.1) again, one arrives at (45). The expressions (37) and (38) for the certainty equivalent and the time-0 value of the optimal payoff under the utility specification of Section 4.1 (saturated CRRA) can be obtained by taking the limits of the expressions above as the subsistence level $\eta$ tends to 0 , or by using (A.3) directly.

Cite this article: Schumacher JM (2021). A note on Gollier's model for a collective pension scheme. Journal of Pension Economics and Finance 20, 187-211. https://doi.org/10.1017/S1474747220000013 